\title{
Evaluation of the effectiveness of treatment with prednisone and azathioprine of autoimmune hepatitis in children
}

\author{
Anna Pniewska ${ }^{1}$, Małgorzata Sobolewska-Pilarczyk ${ }^{1,2}$, Małgorzata Pawłowska ${ }^{1,2}$ \\ ${ }^{1}$ T. Browicz Provincial Infectious Diseases Hospital, Bydgoszcz, Poland \\ 2Department of Children's Infectious Diseases and Hepatology, Collegium Medicum in Bydgoszcz, Nicolaus Copernicus \\ University in Torun, Poland
}

Prz Gastroenterol 2016; 11 (1): 18-23

DOI: $10.5114 / p g .2015 .52566$

Key words: autoimmune hepatitis, azathioprine, prednisone.

Address for correspondence: Anna Pniewska MD, T. Browicz Provincial Infectious Diseases Hospital, 12 Saint Florian 12 St, 85-030 Bydgoszcz, Poland, phone: +48 5232556 16, e-mail: an.pn1@wp.pl

\begin{abstract}
Introduction: Autoimmune hepatitis is rarely diagnosed in children, but the course of the disease is often aggresive. Combination therapy with prednisone and azathioprine improves the prognosis of patients.

Aim: To evaluate the effectiveness of combination therapy with prednisone and azathioprine of autoimmune hepatitis (AlH) in children.

Material and methods: There was a retrospective analysis of the medical records of 15 patients with AlH, diagnosed before18 years of age, treated in the Provincial Infectious Diseases Hospital in Bydgoszcz in the years 2002 to 2013. We analysed the results of laboratory tests, ultrasound examination, endoscopy, and morphological liver pictures, as well as periods of exacerbation of inflammation and side effects of therapy.

Results: Biochemical remission of the disease was achieved on average after 36 days of treatment. Histopathological regression in the control liver biopsy was found in 7/15 patients and progression in 2/15 patients. In the study group 10/15 patients experienced exacerbation of the disease from 1 to 3 times during observation, with an increase of ALT activity to greater than 3 norm, and the remaining 5/15 patients had no increase of ALT activity. In total, 10 patients in the study group experienced 17 exacerbations. In 13/17 cases of exacerbations they were associated with a reduction in the dose of immunosuppressive drugs. There was no correlation between the biochemical exacerbation and changes in the histopathological image. Steroidside effects occurred in 14/15 patients.

Conclusions: The treatment allows for biochemical remission of the disease and significantly improves the prognosis of most patients. However, significant side effects of treatment indicate the need for further exploration of effective and safe therapy, especially in the paediatric population.
\end{abstract}

\section{Introduction}

Autoimmune hepatitis $(\mathrm{AlH})$ is a chronic inflammatory-necrotic process of liver tissue of unknown aetiology. The course of the disease is often aggressive, leading to liver cirrhosis. Genetically determined predisposition to the formation of autoantibodies against antigens of liver cells, and environmental factors (such as infectious and toxic factors, drugs), are taken into account in the pathogenesis of the disease. The incidence of $\mathrm{AlH}$ in Northern Europe is estimated at 1.9 cases per 100000 people per year, with the vast majority being female $(4: 1)$ [1]. Autoimmune hepatitis can occur at any age but is more frequent in young people (under 40 years old). The International AlH Group has developed the AlH scoring system (two versions: a comprehensive system and a simplified system) to help in the diagnosis of AlH $[2,3]$. Elevated $\gamma$-globulin and IgG levels are usually found as well as the presence of autoantibodies (ANA, SMA, LKM 1, anti-LC 1, anti-SLA, ANCA, and anti-LSP) in the blood serum of patients. An antibody titre above $1: 80$ is necessary to confirm the diagnosis in adult patients. Autoimmune hepatitis type 1 is characterised by the presence of ANA or SMA, and type 2 by the presence of LKM-1. In the histopathological im- 
age of liver biopsies in patients with AlH the presence of inflammatory cells in the periportal spaces (chronic septal inflammation, piecemeal necrosis) is usually found, but it is not a pathognomonic image. Rheumatoid arthritis, inflammatory bowel disease, autoimmune thyroiditis, diabetes, vitiligo, alopecia areata, glomerular nephritis, haemolytic anaemia, inflammation of the iris, and polyarteritis nodosa are the most frequently mentioned autoimmune diseases associated with $\mathrm{AlH}$. The occurrence of those diseases is also significantly more frequently found in the family history of the patients than in the general population. Combination therapy with glucocorticoids (usually prednisone) and azathioprine is typically used in the treatment of patients with $\mathrm{AlH}$. There are no clear recommendations that define the duration of the maintenance therapy, but most authors are of the opinion that it should not be less than 2 years, and the decision regarding discontinuation of treatment in patients with biochemical remission of the disease should be preceded by a control liver biopsy [4-7]. The therapy significantly improves the prognosis but does not protect against the progress of liver cirrhosis. Cyclosporine therapy is the alternative in the group of patients with progression of the disease. In the case of progressive cirrhosis, patients are qualified for liver transplantation.

The incidence of autoimmune hepatitis in patients under 18 years of age in the Polish population is estimated at 1/100000/year, with the vast majority being females [7]. The diagnostic criteria of the disease are similar to those used in adults. However, there are some differences. There is moreover no clear opinion on the usefulness of the AlH scoring system in the paediatric population [8-11]. There are also differences in the interpretation of laboratory tests results, e.g. the presence of autoantibodies in blood serum is diagnostic for adult patients in titres above $1: 80$, whereas in children it is already above $1: 20$. Autoimmune hepatitis is rarely diagnosed in children, but due to the significant changes in liver tissue observed at diagnosis and its aggressive course, often leading to liver cirrhosis, AlH is an important clinical problem. Additional problems include the influence of chronic liver disease and long-term therapy for the child's development, growth, and maturation, and often a lack of patient cooperation, resulting from the psychological aspects of puberty.

\section{Aim}

The aim of this study is to evaluate the effectiveness and safety of prednisone and azathioprine therapy in paediatric patients.

\section{Material and methods}

Autoimmune hepatitis was diagnosed in 15 children treated in the Provincial Infectious Diseases Hospital in Bydgoszcz in the years 2002-2013. They were the only female patients, diagnosed at the age between 8 to 17 years (average age: 13 years and 9 months). The time of observation of patients ranged from 9 months to 11 years (mean follow up was 4 years and 8 months). In the cases of 5/15 patients the diagnosis was accidental (the reason for the diagnosis was the accidental discovery increased ALT activity, in the absence of clinical signs), in other cases the most common symptoms were weakness, drowsiness, headache, and abdominal pain. On physical examination yellowish discoloration of the skin and the sclera was found in 8/15 patients, hepatosplenomegaly in $7 / 15$, peripheral oedema in $3 / 15$, and ascites in 1 case. Laboratory abnormalities were found in all the girls (Table I). Positive autoantibody titres were found in $12 / 15$ patients. SMA was found in $7 / 15$ of the girls, ANA in 3/15, and anti-LKM1 in 7/15. Type 1 of the disease was diagnosed in 5/12 patients and type 2 in 3/12; 4/12 patients revealed the presence of antibodies specific to both 1 and 2 type of the disease. Infection with hepatitis $A, B$, and $C$ virus, as well as cytomeg-

Table I. Baseline characteristic of the patients - results of laboratory tests

\begin{tabular}{|c|c|c|c|c|}
\hline Variable & Minimum value & Maximum value & Average value & Percentage of abnormal \\
\hline $\begin{array}{l}\text { ALT activity } \\
(\mathrm{N}: \leq 33 \mathrm{U} / \mathrm{l})\end{array}$ & $73 \mathrm{U} / \mathrm{l}$ & $1890 \mathrm{U} / \mathrm{I}$ & $700 \mathrm{U} / \mathrm{l}$ & 100 \\
\hline $\begin{array}{l}\text { GGT activity } \\
(\mathrm{N}: 5-36 \mathrm{U} / \mathrm{l})\end{array}$ & $31 \mathrm{U} / 1$ & $350 \mathrm{U} / \mathrm{l}$ & $133 \mathrm{U} / \mathrm{l}$ & 87 \\
\hline $\begin{array}{l}\text { Total bilirubin level } \\
(\mathrm{N}: \leq 1.0 \mathrm{mg} / \mathrm{dl})\end{array}$ & $0.41 \mathrm{mg} / \mathrm{dl}$ & $10.28 \mathrm{mg} / \mathrm{dl}$ & $3.73 \mathrm{mg} / \mathrm{dl}$ & 73 \\
\hline $\begin{array}{l}\text { Direct bilirubin level } \\
(\mathrm{N}: \leq 0.2 \mathrm{mg} / \mathrm{dl})\end{array}$ & $0.17 \mathrm{mg} / \mathrm{dl}$ & $6.23 \mathrm{mg} / \mathrm{dl}$ & $2.92 \mathrm{mg} / \mathrm{dl}$ & 93 \\
\hline $\begin{array}{l}\gamma \text {-Globulin level } \\
(\mathrm{N}: 0.8-1.35 \mathrm{~g} / \mathrm{l})\end{array}$ & $1.48 \mathrm{~g} / \mathrm{l}$ & $5.9 \mathrm{~g} / \mathrm{l}$ & $3.18 \mathrm{~g} / \mathrm{l}$ & 100 \\
\hline $\begin{array}{l}\text { IgG level } \\
(\mathrm{N}: 7.0-16.0 \mathrm{~g} / \mathrm{l})\end{array}$ & $14.7 \mathrm{~g} / \mathrm{l}$ & $65.93 \mathrm{~g} / 1$ & $32.18 \mathrm{~g} / 1$ & 87 \\
\hline
\end{tabular}


alovirus, Epstein-Barr virus, and HIV were excluded in all patients. Ultrasound image of the liver was normal in 6/15 cases, non-specific lesions (hepatomegaly, increased echogenicity of the liver tissue) were found in $3 / 15$, and features of advanced cirrhotic remodelling in $6 / 15$. Features of portal hypertension in endoscopy or ultrasound Doppler were found in 5/15 patients. Oesophageal varices stage 1 without any signs of threatening of bleeding were described in endoscopy in 3 patients. In all patients a liver biopsy was performed and inflammatory activity and fibrosis were assessed by a modified Scheuer's scale [6]. Inflammatory activity was estimated at stage 1 to 2 in 7/15 patients, and at stage 3 to 4 in the remaining $8 / 15$. The advancement of fibrosis in 6/15 patients was assessed at 0-2 and in the remaining $9 / 15$ at $3-4$. In 4/15 patients liver cirrhosis was diagnosed (Figure 1).

The patients were treated with combination therapy with prednisone (initial dose $1 \mathrm{mg} / \mathrm{kg}$ body weight/ $24 \mathrm{~h}$, a gradual reduction of the dose was initiated on average after 21 days of treatment until achievement maintenance dose of 5-10 mg/24 h) and azathioprine (at a dose 1-2 mg/kg body weight/24 h).

In our study we conducted a retrospective analysis of medical records of patients diagnosed with $\mathrm{AlH}$, with standard of care intervention. We analysed the effectiveness of treatment in obtaining biochemical remission of the disease, the time to achieve remission (before or after 30 days from the date of the start of treatment), control liver biopsy results, the occurrence of biochemical exacerbations of the disease during the first 3 years of diagnosis, the impact of baseline characteristics of the patients for the time to achieve remission, and the number of exacerbations. We also analysed the frequency and type of therapy side effects.

Biochemical remission of the disease was defined as normalisation of ALT activity and bilirubin and $\gamma$-glob-

A

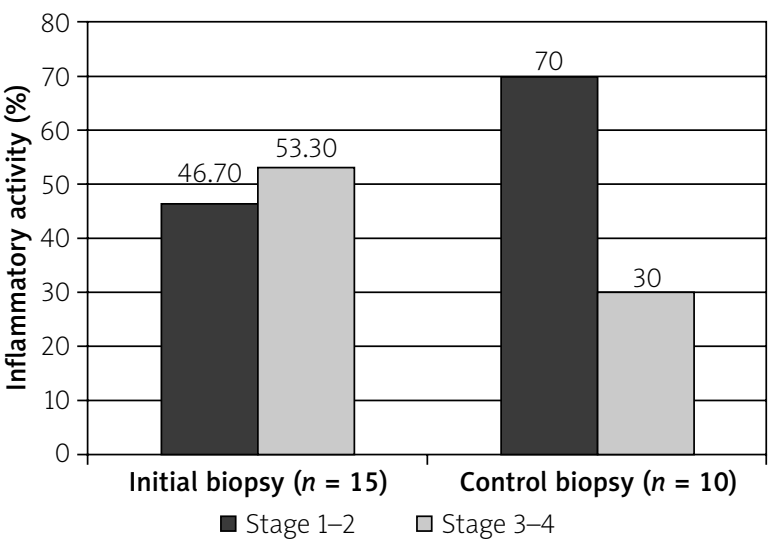

Figure 1. A, B. Liver biopsy results ulin levels, and biochemical exacerbation was defined as an increase in ALT activity to greater than three times the upper normal range. Side effects of treatment included immediate or long-term adverse effects of steroids and azathioprine other than that intended, including cosmetic defects, obesity, osteoporosis, glucose metabolism disorders, hyperlipidaemia, arterial hypertension, growth disorders, haematological abnormalities, and mental and emotional disorders.

\section{Results}

All patients achieved biochemical remission of the disease. It was observed an average of 36 days of treatment (in $46 \%$ in less than 30 days and in 54\% - over 30 days, maximum 80 days). Patients with lower initial ALT activity (less than $500 \mathrm{U} / \mathrm{l}$ ) reached remission within 30 days nearly two times more frequently than patients with ALT activity above 500 (0.63 vs. 0.33). In the study group 5/15 patients had no increase in ALT activity, and the remaining (10/15) experienced biochemical exacerbation of the disease. First exacerbation was observed in 3/10 patients within 12 months and in the remaining $7 / 10$ - within 3 years after the diagnosis of $\mathrm{AlH}$ (mean after 14 months). Five out of 10 patients experienced biochemical exacerbation only once, 3/10 - twice, and 2/10 - three times during follow-up. In total, 17 aggravations of the disease were observed in the group. In $5 / 17$ cases of these incidents, increased total and direct bilirubin levels were found, and in 9/17 - increased $\gamma$-globulin and IgG levels, but the course of exacerbation in individual patients was not similar (an increase in ALT activity was observed both with an increase in the level of bilirubin and $\gamma$-globulin and without it in the same child) (Figure 2). In 13/17 cases of aggravations they were associated with a reduction in the dose of immunosuppressive drugs during maintenance therapy; 3/17 occurred from 4 months to 6 years after discontinua-

\section{B}

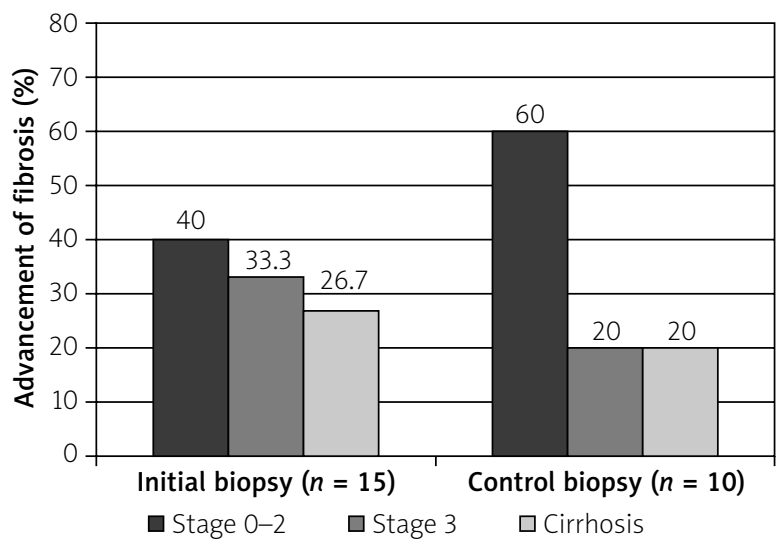




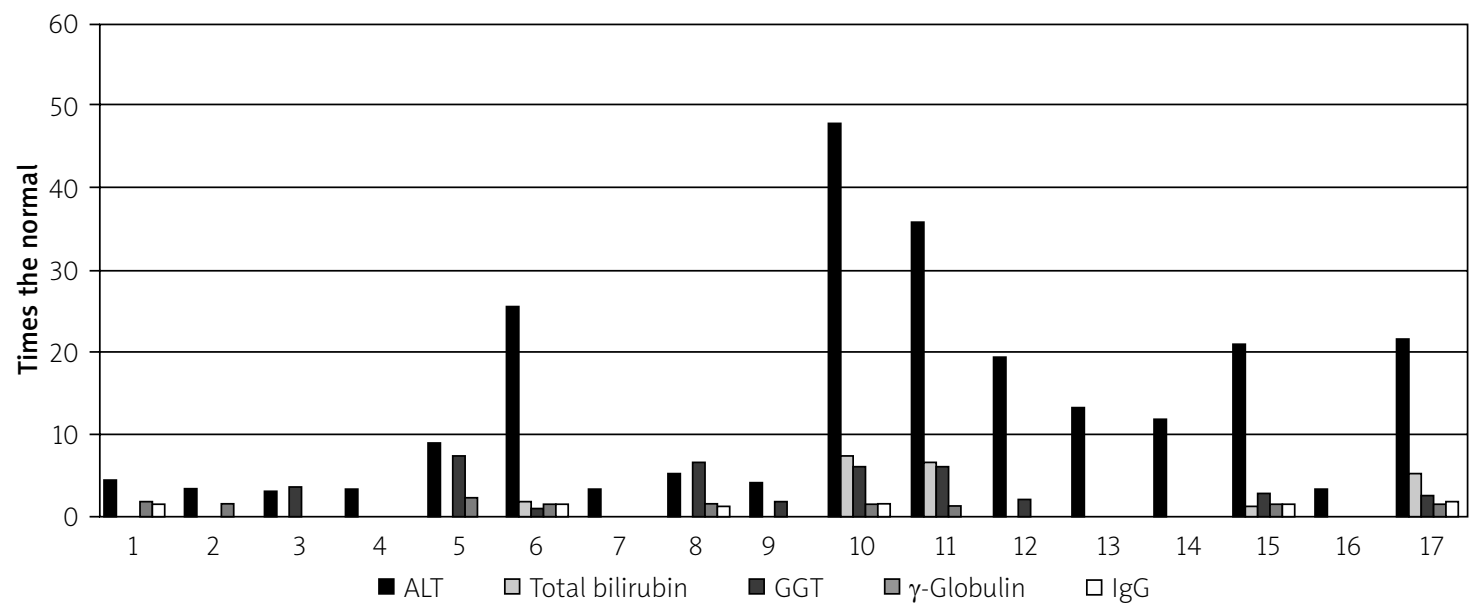

Figure 2. The course of exacerbations - laboratory tests results

tion of treatment (in 2 cases alack of cooperation from the patient was the reason for interruption of therapy - the patient discontinued treatment without consultation and did not report on the visits to the clinic). One case of exacerbation was associated with pregnancy, during which the patient received prednisone monotherapy only. There were no relationships between clinical outcome and incidences of aggravations and age at diagnosis, serum $\gamma$-globulin and IgG levels, and the presence and type of autoantibodies. The number of exacerbations in the first 3 years was correlated with the ALT activity at the time of diagnosis. Two or more biochemical exacerbations during follow-up were observed significantly more frequently in the group of patients in whom ALT activity at the time of diagnosis was greater than $500 \mathrm{U} / \mathrm{l}(n=6$; ALT activity 686-1890 U/l, mean: $1355 \mathrm{U} / \mathrm{l})$ compared to patients with ALT activity below $500 \mathrm{U} / \mathrm{l}(n=7$; ALT activity 89-466 U/l, mean: $221 \mathrm{U} / \mathrm{l})$ ( 0.83 vs. 0.14 , hazard ratio $=5.93)$. However, the study group was too small to clearly assess the impact of this parameter on the further course of the disease. Patients without identified exacerbations but with a follow-up of less than 3 years were excluded from this analysis (2/15).

Control liver biopsy was performed in 10 patients. Regression of inflammation and fibrosis was found in 7/10 patients (on average, 34 months after the initiating of treatment), and histopathological progression in 2/10 (Figure 1). There was no correlation between the biochemical exacerbations of the disease and changes in the histopathological image. In the group with histopathological regression, biochemical aggravations were not observed in $2 / 7$ patients, and in the remaining 5/7 - from 1 to 3 aggravations (in total, 13 cases of biochemical exacerbations were observed in these groups, with an increase in $\gamma$-globulin and IgG levels in 5/13 cases). Among patients with histopathological progression, in one case there was no biochemical exacerbation observed, and in the second there were two exacerbations with increased ALT activity and $\gamma$-globulin level, without an increase of bilirubin level. One of those 2 patients presented significant progression of the disease despite the treatment. In this patient type $1 \mathrm{AlH}$ was diagnosed (the presence of ANA, but in low titre). Baseline ALT activity was $248 \mathrm{U} / \mathrm{l}$, total bilirubin $2.49 \mathrm{mg} / \mathrm{dl}, \gamma$-globulin - $3.04 \mathrm{~g} / \mathrm{l}$, IgG - $33.2 \mathrm{~g} / \mathrm{l}$. She reached biochemical remission of the disease 80 days after treatment initiation; there was no biochemical exacerbation observed, but thrombocytopenia (3344 000/UI), leukopaenia (2900-4400/UI), and abnormalities in the coagulation (prothrombin index: 66-81\%) were chronic problems. In the initial liver biopsy inflammatory activity was estimated at stage 3 , also fibrosis at stage 3, and in control liver biopsy there was histopathological progression to cirrhosis, and in endoscopy oesophageal varices were found.

The presence of significant comorbidities and their possible impact on the course of $\mathrm{AlH}$ and the effectiveness of treatment were also analysed. In the study group inflammatory bowel disease was diagnosed in $3 / 15$ patients, and in 2/15 - autoimmune thyroiditis. No significant differences in the course of $\mathrm{AlH}$ in these patients were found compared to the patients without comorbidities.

The safety profile of the treatment was another aspect of the study. The side effects of the therapy were observed in all patients. Steroids side effects in the form of cosmetic defects were observed with varying intensity in $14 / 15$ patients, and they were the most common side effects of the treatment. In 5/14 cases they were significant. Those patients had experienced exacerbations more frequently - two or three 
times. The occurrence of aggravations was the cause of prolonged use of high doses of prednisone in those patients, which resulted in increased side effects of steroids: weight gain (obesity in 5 patients), abnormal distribution of subcutaneous fat, stretch marks, acne, and hirsutism. Given the fact that the patients were in adolescence, cosmetic defects were an important psychological problem. Depressive disorders caused the necessity to include drug treatment in 1 case, and emotional disorders that caused a lack of patient cooperation in two cases. In addition, hyperlipidaemia (2/15), anaemia (1/15), and osteoporosis (recognised based on bone density scanning) (1/15) were observed. In the study group there were no identified glucose metabolism disorders, arterial hypertension, or growth disorders.

\section{Discussion}

Combination therapy with prednisone and azathioprine is the most commonly used for patients with $\mathrm{AlH}$, in both adults and children. This therapy is highly effective in achieving biochemical remission of the disease, and it significantly improves the prognosis of patients; however, it does not protect against progression of liver cirrhosis. In our observations, such combination therapy led to the achievement of biochemical remission of the disease in all patients. However, in 2/15 patients histological progression was observed. Qualification for a liver transplant is currently being considered in 1 of our patients due to significant progression of the disease.

Side effects of treatment are a major problem, especially in the paediatric population. The incidence of major steroid side effects, resulting in discontinuation of therapy, is estimated at $13 \%$. Cosmetic defects and obesity occur in $47 \%$ of patients, osteoporosis in $27 \%$, and disorders of glucose metabolism in $20 \%$ [12]. In our observations cosmetic defects were observed in $93 \%$ patients, obesity in 33\%, osteoporosis in $7 \%$, and hyperlipidaemia in $13 \%$. We did not observe glucose metabolism disorders, arterial hypertension, or growth disorders.

There have been reports of the positive effects of budesonide used instead of prednisone in the treatment of $\mathrm{AlH}$. Manns et al. conducted an international, randomised, double-blind trial with the participation of a Polish centre, evaluating the usefulness of budesonide in achieving and maintaining clinical remission in patients with AlH [13]. According to the authors, the use of budesonide in combination with azathioprine in patients with $\mathrm{AlH}$ without liver cirrhosis was associated with higher rates of achieving full biochemical remission and fewer side effects of steroids, compared to prednisone. Woynarowski et al. did not confirm these findings in children [14]. In our observations, 1 girl received modified treatment during maintenance therapy - she received budesonide instead of prednisone because of severe side effects of steroids. The treatment was effective in maintaining biochemical remission of the disease. There was also a slight reduction in the severity of side effects of steroids.

\section{Conclusions}

In our study the treatment of $\mathrm{AlH}$ with prednisone and azathioprine was effective in achieving biochemical remission of the disease, but therapy does not always protect against the development of liver cirrhosis. Exacerbations of inflammation were observed most often after prednisone dose reduction. There was no correlation between the biochemical exacerbations and changes in the histopathological image. Cosmetic defects were the most common side effects of the therapy.

\section{Conflict of interest}

The authors declare no conflict of interest.

\section{References}

1. Czaja AJ, Freese DK. Diagnosis and treatment of autoimmune hepatitis. Hepatology 2002; 36: 479-97.

2. Alvarez F, Berg PA, Bianchi FB, et al. International Autoimmune Hepatitis Group Report: review of criteria for diagnosis of autoimmune hepatitis. J Hepatol 1999; 31: 929-38.

3. Hennes EM, Zeniya M, Czaja AJ, et al. Simplified criteria for the diagnosis of autoimmune hepatitis. Hepatology 2008; 48 : 169-76.

4. Czaja AJ. Difficult treatment decisions in autoimmune hepatitis. World J Gastroenterol 2010; 16: 934-47.

5. Czaja AJ. Advances in the current treatment of autoimmune hepatitis. Dig Dis Sci 2012; 57: 1996-2010.

6. Oo YH, Hubscher SG, Adams DH. Autoimmune hepatitis: new paradigms in the pathogenesis, diagnosis, and management. Hepatol Int 2010; 4: 475-93.

7. Woźniak M, Woynarowski M, Socha J. The guidelines for autoimmune hepatitis therapy in children treated in Children's Health Memorial Institute. Medical Science Review. Hepatology 2009; 9: 12-9.

8. Mileti E, Rosenthal P, Peters MG. Validation and modification of simplified diagnostic criteria for autoimmune hepatitis in children. Clin Gastroenterol Hepatol 2012; 10: 417-21.e1-2.

9. Hiejima E, Komatsu H, Sogo T, et al. Utility of simplified criteria for the diagnosis of autoimmune hepatitis in children. J Pediatr Gastroenterol Nutr 2011; 52: 470-3.

10. Ferri PM, Ferreira AR, Miranda DM, et al. Diagnostic criteria for autoimmune hepatitis in children: a challenge for pediatric hepatologists. World J Gastroenterol 2012; 18: 4470-3.

11. Desmet VJ, Gerber M, Hoofnagle JH, et al. Classification of chronic hepatitis: diagnosis, staging and grading. Hepatology 1994; 19: 1513-20. 
12. Manns MP, Czaja AJ, Gorham JD, et al. Diagnosis and management of autoimmune hepatitis. Hepatology 2010; 51 2193-213.

13. Manns MP, Woynarowski M, Kreisel W, et al. Budesonide induces remission more effectively than prednisone in a controlled trial of patients with autoimmune hepatitis. Gastroenterology 2010; 139: 1198-206.

14. Woynarowski M, Antal N, Baruch Y, et al. Budesonide versus prednisone with azathioprine for the treatment of autoimmune hepatitis in children and adolescents. J Pediatr 2013; 163: 1347-53.

Received: 1.12.2014

Accepted: 26.02 .2015 